\title{
PHIlebreglet
}

Servicio de Publicaciones y Difusión Científica

Philologica Canariensia 21 (2015), 109-132 eISSN: 2386-8635

DOI: 10.20420/PhilCan.2015.0036

\section{QUIZÁ(S) Y TAL VEZ EN NOVELISTAS DE ESPAÑA Y AMÉRICA}

\author{
MAGNOLIA TROYA DÉNIZ \\ Universidad de Las Palmas de Gran Canaria
}

\section{RESUMEN}

En este artículo se examina la frecuencia de quizá(s) y tal vez en textos literarios narrativos de ocho novelistas procedentes de España y América. El objetivo es comprobar estadísticamente si el autor es una variable que condiciona la aparición de estos adverbios de duda. Los datos proceden del análisis de 3476 ocurrencias que han sido extraídas de un corpus de 32 novelas publicadas entre 2001 y 2014. Los resultados no solo revelan que la interacción de la variable autor con el empleo de quizá(s) y tal vez es significativa, sino que también hay asociación entre las variables en diferentes contextos. Así pues, el estudio permite confirmar que el uso de los mencionados adverbios es un indicador de los hábitos lingüísticos del escritor.

PALABRAS ClAVE: quizá(s), tal vez, variación idiolectal, narrativa, español, estilometría

Quizá(s) and tal vez in Some Spanish and Latin American Authors

\begin{abstract}
In this paper I intend to analyze the rate of occurrence of two adverbials of possibility, quizá(s) and tal vez, in prose narratives by eight Spanish and Latin American novelists. The main goal is to check, through a thorough statistical scrutiny, whether these adverbs are author-related or not. The data, a total of 3476 instances to be sampled, are drawn from an overall corpus of 32 novels published between 2001 and 2014. The results yielded by such analysis not only show that the presence of these adverbs is somewhat determined by the author but also that the variables are interrelated across different contexts. Thus, what the ensuing lines come to prove is that the use of such adverbs of possibility is a marker of the writer's idiosyncratic style.
\end{abstract}

KEYWORDS: quizá(s), tal vez, idiolectal variation, narrative, Spanish, stylometry 


\section{INTRODUCCIÓN}

Las formas quizá(s) y tal vez, incluidas en la nómina de los tradicionalmente denominados adverbios y locuciones adverbiales de duda, funcionan como operadores de modalidad epistémica. Como se sabe, la modalidad es un tema que ha despertado un notable interés en las investigaciones lingüísticas, tal y como lo demuestra el importante número de trabajos en los que se trata este aspecto. ${ }^{1}$ A partir de la formulación del concepto de modalité que hace Bally (1932), la modalidad se ha considerado, de manera general, como "la expresión gramatical de la actitud del sujeto hablante (o de otro sujeto pensante) ante el contenido de la enunciación, especialmente de su núcleo predicativo" (Zavadil, 1968, 58). ${ }^{2}$ Kovacci (1999, 755) indica que quizá(s) y tal vez son adverbios relacionados con la modalidad que expresan actitud dubitativa -indicadores o reforzadores de actitud3 3 .

En cuanto a los tipos de modalidad, Otaola Olano (1988, 101-102) distingue las modalidades de la enunciación, que "expresan las diversas actitudes del hablante ante el oyente", y las modalidades del enunciado, que "manifiestan la actitud del hablante ante el enunciado o mensaje". Los adverbios quizá(s) y tal vez se encuentran entre los medios de los que disponen los hablantes para expresar la modalidad del enunciado. Concretamente, se trata de adverbios asertivos o modificadores de la aserción que expresan modalidades lógicas relacionadas con lo posible (Otaola Olano, 1988, 108).4

Ridruejo (1999, 3214), sin embargo, considera que resulta más útil para la caracterización de las categorías gramaticales en español la distinción que se establece entre modalidad deóntica y modalidad epistémica (Lyons, 1977; Perkins, 1983; Palmer, 1986). Según Perkins (1983), la posibilidad y la necesidad, esto es, los dos grandes ejes en los que se articula la modalidad (Lyons, 1977, 787), están condicionadas por leyes sociales (permiso u obligación) en la modalidad deóntica y por leyes racionales relacionadas con juicios personales en la modalidad epistémica. Los adverbios quizá(s) y tal vez son recursos lingüísticos con los que se manifiesta este último tipo de modalidad. En palabras de Ridruejo (1999, 3214), la modalidad epistémica "se define como la expresión del grado de compromiso que el hablante asume con respecto a la verdad de la proposición contenida en un enunciado". También, la Nueva gramática de la lengua española 
(RAEyAALE, 2009, 2350-2351) inscribe los adverbios de duda en el grupo de los modales epistémicos ya que denotan "la posibilidad de que se dé una determinada situación".

En cuanto a los trabajos en los que se cuantifica el número de ocurrencias de quizá(s) y tal vez, cabe mencionar los estudios que realizan Woehr (1972) y Bauhr (1990).

Woehr (1972) determina la frecuencia de quizá(s) y tal vez a partir del análisis de un corpus de fragmentos seleccionados en 100 textos (30 obras de teatro, 30 novelas, 20 ensayos y 20 artículos periodísticos) que fueron publicados entre 1942 y 1969 y escritos por autores nacidos en España y América. Si bien los resultados generales muestran que quizá(s) posee un índice de frecuencia mayor que tal vez (176 ocurrencias vs. 122), la locución adverbial es más común en el español americano (89 casos vs. 77) que en el español peninsular (33 ejemplos vs. 99).

Por su parte, Bauhr (1990) constata en 11 novelas de distintos escritores españoles publicadas entre 1951 y 1971 que el porcentaje correspondiente a tal vez (51.9\%) supera en 3.8 puntos al de quizá(s). ${ }^{6}$ Asimismo, en sus conclusiones apunta que la elección del adverbio puede ser "un hecho regional" puesto que los novelistas del centro de España usan más quizá y los catalanes prefieren tal vez. No obstante, en su opinión se trataría de un "hecho irreflexivo, automático, que deja en el texto una parte de las 'huellas digitales' del autor" (Bauhr, 1990, 192).7 De acuerdo con esto, la elección de quizá(s) y tal vez sería un indicador de los hábitos lingüísticos del escritor.

En este artículo nos proponemos ir más allá de los datos cuantitativos para comprobar, a través del análisis estadístico, si el uso de quizá(s) y tal vez está condicionado por el autor. Además de los datos generales que se desprendan de la interacción entre adverbio y autor, también examinaremos el comportamiento de estas variables en distintos contextos. De igual modo, estableceremos cuál es la intensidad de la asociación y en qué escritores las diferencias de frecuencias entre quizá(s) y tal vez resultan significativas. Así pues, desde una perspectiva estilométrica, los resultados de este estudio contribuirán a determinar si el empleo de los mencionados adverbios puede ser un parámetro significativo que, junto a otros rasgos idiolectales, permita caracterizar la forma de expresarse de un individuo. 


\section{Metodología}

Para la obtención de los datos nos hemos servido de un corpus de textos literarios narrativos pertenecientes a 8 escritores que proceden de España y América: Almudena Grandes, Rosa Ribas, Javier Marías, Eduardo Mendoza, Isabel Allende, Florencia Bonelli, Eduardo Sacheri y Mario Vargas Llosa. De cada autor hemos seleccionado 4 novelas publicadas entre 2001 y 2014. Así pues, hemos trabajado con 32 obras de cuyas ediciones digitales en formato EPUB hemos extraído todos los enunciados en los que aparecían quizá(s) y tal vez. ${ }^{8}$ En total hemos encontrado 3476 ocurrencias de estos adverbios. Con la finalidad de poder llevar a cabo el procesamiento de los datos, hemos codificado cada uno de estos casos en función de las variables consideradas.

En lo que respecta al tratamiento estadístico, hemos realizado con el programa SPSS, versión 19, un análisis de contingencia con el objeto de determinar si existen asociaciones entre las variables cualitativas. El test empleado para conocer la relación entre las variables es el estadístico chi-cuadrado de Pearson $\left(\chi^{2}\right) .{ }^{9}$ Una vez asumida la dependencia entre las variables, el cálculo de los residuos tipificados corregidos nos ha permitido conocer qué factores contribuyen de manera significativa al valor del estadístico de contraste. ${ }^{10}$ Por último, para calcular el grado de fortaleza de las asociaciones hemos utilizado el coeficiente V de Cramer (V). ${ }^{11}$

\section{RESUltados GENERALES}

3.1. El gráfico 1 recoge la distribución porcentual de las 3476 ocurrencias de quizá(s) y tal vez que hemos documentado. 


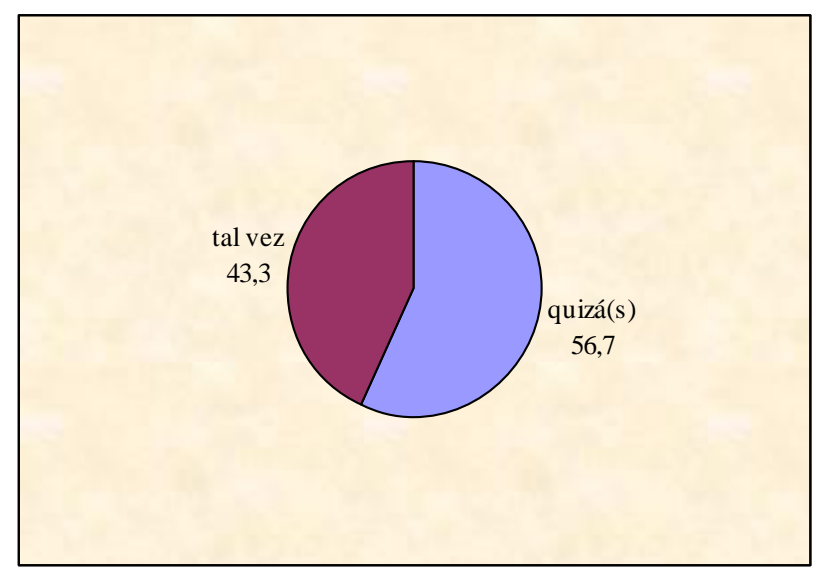

Gráfico 1. Porcentajes de quizá(s) y tal vez en la muestra.

Nuestros resultados coinciden con los que aporta Woehr (1972): quizá(s) es el adverbio que obtiene un porcentaje más elevado; su índice se incrementa 13.4 puntos con respecto a la locución tal vez. De igual modo, el análisis de los datos según la procedencia del novelista (tabla 1) corrobora las tendencias que anota Woehr (1972): los escritores españoles usan con mayor frecuencia (65.3\%) el adverbio quizá(s), mientras que los americanos propician la aparición de tal vez $(70 \%) .{ }^{12}$

\begin{tabular}{|c|c|c|c|c|c|}
\hline \multirow{2}{*}{ procedencia } & \multirow{2}{*}{$\mathrm{N}$} & \multicolumn{2}{|c|}{ quizá(s) } & \multicolumn{2}{|c|}{ tal vez } \\
\hline & & $\%$ & RTC & $\%$ & RTC \\
\hline España & 2636 & $65 \cdot 3$ & 18 & 34.7 & -18 \\
\hline América & 840 & 30 & -18 & 70 & 18 \\
\hline Total & 3476 & 56.7 & & $43 \cdot 3$ & \\
\hline
\end{tabular}

Tabla 1. Quizá(s) y tal vez * procedencia de los autores.

La prueba chi-cuadrado determina que la procedencia de los autores es una variable que condiciona el uso de quizá(s) y tal vez ( $\mathrm{p}=.000)$. Asimismo, los residuos tipificados corregidos $( \pm 18)$ indican que todas las asociaciones contribuyen de manera muy significativa al valor del estadístico de contraste. ${ }^{13}$ 
Por otro lado, la probabilidad asociada a la prueba chi-cuadrado también resulta inferior a .05 ( $\mathrm{p}=.000)$ cuando se examina el reparto de las formas adverbiales en función de los autores (tabla 2). Sin embargo, el coeficiente de Cramer revela que hay una relación de intensidad más alta entre estas variables que la observada entre los adverbios y la procedencia geográfica del autor $(\mathrm{V}=.497$ vs. $\mathrm{V}=.305)$. De acuerdo con esto, las preferencias idiolectales tienen mayor incidencia en la elección de quizá(s) y tal vez.

\begin{tabular}{|l|c|c|c|c|c|}
\hline \multirow{2}{*}{ autores } & \multirow{2}{*}{$\mathrm{N}$} & \multicolumn{2}{c|}{ quizá(s) } & \multicolumn{2}{c|}{ tal vez } \\
\cline { 3 - 6 } & & $\%$ & $\mathrm{RTC}$ & $\%$ & $\mathrm{RTC}$ \\
\hline Almudena Grandes & 627 & 63.3 & 3.7 & 36.7 & -3.7 \\
\hline Rosa Ribas & 418 & 63.2 & 2.8 & 36.8 & -2.8 \\
\hline Javier Marías & $\begin{array}{c}132 \\
9\end{array}$ & 69.4 & 11.8 & 30.6 & -11.8 \\
\hline Eduardo Mendoza & 262 & 52.3 & -1.5 & 47.7 & 1.5 \\
\hline Isabel Allende & 153 & .7 & -14.3 & 99.3 & 14.3 \\
\hline Florencia Bonelli & 256 & 87.9 & 10.5 & 12.1 & -10.5 \\
\hline Eduardo Sacheri & 187 & 2.1 & -15.5 & 97.9 & 15.5 \\
\hline Mario Vargas Llosa & 244 & 9 & -15.6 & 91 & 15.6 \\
\hline \multicolumn{2}{|c|}{ Total } & 347 \\
6 & 56.7 & & 43.3 & \\
\hline $\begin{array}{l}\chi^{2}=857.395, \text { gl=7; } \mathrm{p}=.000 \\
\text { V=.497 }\end{array}$ & & & & \\
\hline
\end{tabular}

Tabla 2. Quizá(s) y tal vez * autores.

La frecuencia de quizá(s) supera a la calculada para tal vez en los cuatro novelistas españoles: Marías destaca con un 69.4\% y un importante residuo tipificado que se sitúa en el 11.8, mientras que Mendoza reduce a 4.6 puntos porcentuales la diferencia con respecto a la locución adverbial y, además, es el único escritor que obtiene un residuo tipificado no significativo ( \pm 1.5 ). Ahora bien, el valor positivo que tiene el residuo correspondiente a tal vez en el autor catalán denota que hay contextos en los que Mendoza, como se irá viendo, favorece el empleo de la locución adverbial. ${ }^{14}$ En cuanto a los resultados que aportan los novelistas americanos, cabe destacar, en primer lugar, que todos los residuos son muy significativos. Además, en Allende, Sacheri y Vargas Llosa se confirma - con porcentajes muy elevados que alcanzan el 99.3\%, 97.9\% y 91\%, respectivamente- la ya mencionada 
tendencia de los escritores americanos a usar la locución con más frecuencia. Por el contrario, en la escritora argentina Bonelli quizá(s) representa el $87.9 \%$ de los ejemplos, valor incluso superior al que tienen los autores españoles.

3.2. La Real Academia Española indica que el adverbio quizá procede del latín qui sapit 'quién sabe' (DRAE, 2014), mientras que quizás se creó por analogía con otros adverbios terminados en $-s$ (DPD, 2005). ${ }^{15}$ Se trata, pues, de dos variantes del mismo adverbio, motivo por el que en este trabajo hemos agrupado sus ocurrencias frente a los casos de tal vez. Pese a ello, no hemos querido prescindir de los resultados más relevantes que se desprenden del análisis de la distribución de quizá y quizás. Los datos del gráfico 2 evidencian que en nuestros materiales hay un claro predominio de la forma etimológica, que representa el $68.7 \%$ de los 1972 ejemplos documentados.

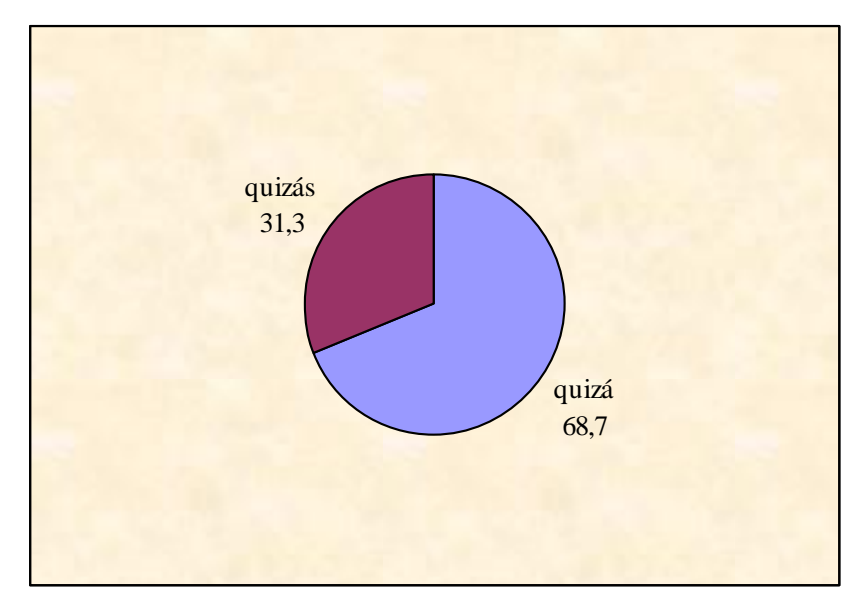

Gráfico 2. Porcentajes de quizá y quizás en la muestra.

En lo que respecta a la interacción de la variable autor con el uso de quizá y quizás (tabla 3), ${ }^{16}$ no solo la prueba chi-cuadrado determina que hay dependencia entre estas variables ( $\mathrm{p}=.000)$, sino que, además, la intensidad de la asociación es muy alta $(\mathrm{V}=.860)$. Como puede verse, los seis escritores contribuyen de manera significativa al valor del estadístico: especialmente elevados son los residuos en Grandes $( \pm 32.9)$ y en Marías ( \pm 28.2$)$. 


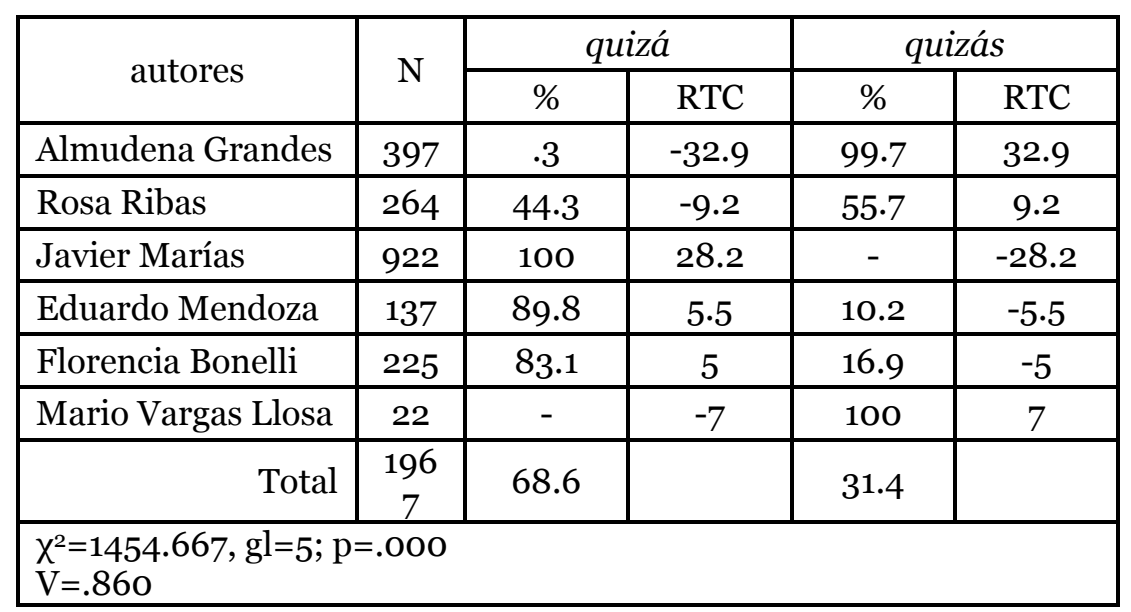

Tabla 3. Quizá y quizás * autores.

Marías solo utiliza la forma etimológica en las 922 ocurrencias que hemos encontrado de este adverbio. También es notable el porcentaje de quizá en Mendoza (89.8\%) y en Bonelli (83.1\%). Frente a esto, quizás es la variante con mayor índice de frecuencia en los tres restantes autores. Grandes tiene un uso prácticamente categórico del adverbio quizás (99.7\%): solo 1 caso de los 397 ejemplos corresponde a quizá. Igualmente, Vargas Llosa solo emplea quizás, aunque el número de ocurrencias se limita a 22. Por último, Ribas es la autora que más alterna la aparición de quizá y quizás: el porcentaje de esta última variante supera al de quizá en 11.4 puntos.

\section{RESULTADOS CONTEXTUALES}

\subsection{Según la función de quizá(s) y tal vez}

De acuerdo con la propuesta que hace Aurová (2013), consideramos que quizá(s) y tal vez no funcionan como operadores oracionales cuando modifican a un constituyente concreto - sintagma nominal, adjetival, adverbial o preposicional- o aparecen como marcadores discursivos entre pausas. Por el contrario, estos adverbios son operadores cuando afectan a toda la oración y, consecuentemente, inciden en el sintagma verbal. A continuación anotamos algunos ejemplos en los que puede observarse el uso de quizá(s) y tal vez con estas funciones: 
(1) a. Todos los animales que rodean a la primigenia pareja están supuestamente vivos y en movimiento o alerta, monos, liebres, pavos, grullas, tejones, quizá un tucán y hasta la serpiente, que asoma con expresión demasiado humana entre las muy verdes hojas del manzano. (Javier Marías, 2011)

b. Además, lo encontró serio y distante, enojado quizá, lo que la angustió sobremanera y, segura de que el enfado se debía a la pequeña escena con Valerie Le Bon deseó no haber abierto la boca. (Florencia Bonelli, 2006)

c. Anthony siente una leve emoción al interpretar, quizás erróneamente, que el teniente coronel se preocupa por su seguridad. (Eduardo Mendoza, 2010)

d. La primera, los hombres trajeados y las mujeres en traje chaqueta que caminaban rápidos, quizás en dirección a la Bolsa o a las oficinas que jalonan Goetheplatz. (Rosa Ribas, 2009)

(2) a. Durante unos segundos, tal vez un minuto larguísimo, los tres respetaron las sorprendentes reglas de aquella escena, y lo único que se movió fue el dedo índice de Alfonso Olmedo, y el vertiginoso torrente de imágenes y sonidos que obedecía a los impulsos de su voluntad en una pantalla que parecía a punto de estallar en pedazos, incapaz de soportar tanta presión. (Almudena Grandes, 2002)

b. El mismo pelo rubio, tal vez un poco más raleado que en nuestro último encuentro. (Eduardo Sacheri, 2009)

c. Guardó en el fondo de su alma el amor por Gambo, resignada a la idea de no verlo en mucho tiempo, tal vez nunca más, pero lo llevaba clavado en el corazón y suponía que se hallaba entre las filas de ese Toussaint. (Isabel Allende, 2009)

d. No alcancé a entender lo que decían, pero estaban jugando a algo, tal vez a las damas, tal vez al Yan-Ken-Po, y, a juzgar por las risitas, la pasaban muy bien. (Mario Vargas Llosa, 2006)

(3) a. Quizás estábamos locos, pero esa locura me impidió entender la historia de Paloma, las palabras de mi abuelo, para vivir aquí, hay cosas que es mejor no saber, incluso no entender ... - y de pronto se extinguieron todas las sonrisas-. (Almudena Grandes, 2007)

b. Había perdido confianza en ese hombre, quizá estuviera protegiendo a Indiana. (Isabel Allende, 2014)

c. Quizá la habían confundido con otra señora Cáceres. (Florencia Bonelli, 2003)

d. Quizás no estaba lejano el día en que pudiera volver a circular por las calles de la ciudad como un transeúnte anónimo. (Mario Vargas Llosa, 2013)

(4) a. Ahora que lo hemos cazado, subirá varios puestos, y tal vez consiga hacerse el amo en caso de que Rimaç no salga de ésta. (Rosa Ribas, 2007)

b. También se miraría la mano sana y tal vez pensaría: 'Qué suerte, al menos esta la tengo intacta'. (Javier Marías, 2007)

c. Tal vez sea cierto lo que nos ha dicho antes y le guiara la intención de matar a Pardalot. (Eduardo Mendoza, 2001)

d. Tal vez la tenga alguna de las vecinas. (Eduardo Sacheri, 2014)

La significación estadística asociada a la prueba chi-cuadrado $(\mathrm{p}=.000)$ pone de manifiesto la dependencia entre el adverbio cuando no se usa como complemento del 
verbo y el autor (tabla 4); en este contexto hay una intensidad de asociación alta entre las variables $(\mathrm{V}=.491)$.

\begin{tabular}{|c|c|c|c|c|c|}
\hline \multirow{2}{*}{ autores } & \multirow{2}{*}{$\mathrm{N}$} & \multicolumn{2}{|c|}{ quizá(s) } & \multicolumn{2}{|c|}{ tal vez } \\
\hline & & $\%$ & RTC & $\%$ & RTC \\
\hline Almudena Grandes & 285 & 67.4 & 3.3 & 32.6 & $-3 \cdot 3$ \\
\hline Rosa Ribas & 111 & 64.9 & 1.3 & 35.1 & -1.3 \\
\hline Javier Marías & 510 & 68.8 & 5.8 & 31.2 & -5.8 \\
\hline Eduardo Mendoza & 111 & 66.7 & 1.7 & 33.3 & -1.7 \\
\hline Isabel Allende & 31 & - & -6.7 & 100 & 6.7 \\
\hline Florencia Bonelli & 104 & 86.5 & 6 & 13.5 & -6 \\
\hline Eduardo Sacheri & 67 & 6 & -9 & 94 & 9 \\
\hline Mario Vargas Llosa & 130 & 8.5 & -12.3 & 91.5 & 12.3 \\
\hline Total & $\begin{array}{c}134 \\
9\end{array}$ & 58.9 & & 41.1 & \\
\hline
\end{tabular}

Tabla 4. Quizá(s) y tal vez cuando no son operadores oracionales * autores.

De igual modo, el análisis estadístico arroja una probabilidad significativa $(\mathrm{p}=.000)$ en el contraste entre las variables adverbio en función de operador oracional y autor (tabla 5); también el coeficiente de Cramer revela que la fortaleza de la relación es alta (V=.508).

\begin{tabular}{|c|c|c|c|c|c|}
\hline \multirow{2}{*}{ autores } & \multirow{2}{*}{$\mathrm{N}$} & \multicolumn{2}{|c|}{ quizá(s) } & \multicolumn{2}{|c|}{ tal vez } \\
\hline & & $\%$ & RTC & $\%$ & RTC \\
\hline Almudena Grandes & 342 & 59.9 & 1.85 & 40.1 & -1.85 \\
\hline Rosa Ribas & 307 & 62.5 & 2.7 & $37 \cdot 5$ & -2.7 \\
\hline Javier Marías & 819 & 69.7 & 10.5 & 30.3 & -10.5 \\
\hline Eduardo Mendoza & 151 & 41.7 & -3.5 & 58.3 & 3.5 \\
\hline Isabel Allende & 122 & .8 & -12.5 & 99.2 & 12.5 \\
\hline Florencia Bonelli & 152 & 88.8 & 8.6 & 11.2 & -8.6 \\
\hline Eduardo Sacheri & 120 & - & -12.6 & 100 & 12.6 \\
\hline Mario Vargas Llosa & 114 & 9.6 & -10.1 & 90.4 & 10.1 \\
\hline Total & $\begin{array}{c}212 \\
7\end{array}$ & $55 \cdot 4$ & & 44.6 & \\
\hline
\end{tabular}

Tabla 5. Quizá(s) y tal vez como operadores oracionales * autores. 
Los residuos tipificados carecen de significación en Mendoza $( \pm 1.7)$ y en Ribas $( \pm 1.3)$ cuando quizá(s) y tal vez no complementan al verbo. Ahora bien, en los contextos en que los adverbios funcionan como operadores oracionales únicamente deja de contribuir al valor del estadístico el residuo que muestra Grandes ( \pm 1.85 ). Por otra parte, los datos de la tabla 5 no solo evidencian una diferencia porcentual significativa en Mendoza $( \pm 3.5)$, sino que también revelan que tal vez es la forma con mayor índice de frecuencia en el autor catalán $(58.3 \%, 13.7$ puntos por encima del porcentaje total).

\subsection{Según la posición de quizá(s) y tal vez con respecto al verbo}

$\mathrm{Al}$ comparar las cifras absolutas que se anotan en la tabla 6 con las que se muestran en la tabla 5, comprobamos que la anteposición de los adverbios al núcleo verbal oracional es la posición que predomina de manera clara en las 32 novelas analizadas: solo 90 casos (4.2\%) de los 2127 ejemplos de quizá(s) y tal vez que funcionan como operadores oracionales aparecen pospuestos al verbo. Por esta razón los resultados correspondientes a la anteposición adverbial (tabla 6) son prácticamente idénticos a los que se obtienen en el examen de los adverbios de duda como complementos verbales (tabla 5).17

\begin{tabular}{|c|c|c|c|c|c|}
\hline \multirow{2}{*}{ autores } & \multirow{2}{*}{$\mathrm{N}$} & \multicolumn{2}{|c|}{ quizá(s) } & \multicolumn{2}{|c|}{ tal vez } \\
\hline & & $\%$ & RTC & $\%$ & RTC \\
\hline Almudena Grandes & 318 & 59.7 & 1.7 & 40.3 & -1.7 \\
\hline Rosa Ribas & 284 & 62 & 2.4 & 38 & -2.4 \\
\hline Javier Marías & 794 & 69.8 & 10.5 & 30.2 & -10.5 \\
\hline Eduardo Mendoza & 151 & 41.7 & -3.5 & 58.3 & 3.5 \\
\hline Isabel Allende & 122 & .8 & -12.5 & 99.2 & 12.5 \\
\hline Florencia Bonelli & 148 & 88.5 & 8.4 & 11.5 & -8.4 \\
\hline Eduardo Sacheri & 113 & - & -12.2 & 100 & 12.2 \\
\hline Mario Vargas Llosa & 107 & 10.3 & -9.6 & 89.7 & 9.6 \\
\hline Total & $\begin{array}{c}203 \\
7\end{array}$ & $55 \cdot 3$ & & 44.7 & \\
\hline
\end{tabular}

Tabla 6. Quizá(s) y tal vez antepuestos al verbo * autores. 
En cuanto a la correlación entre adverbio y autor en el contexto de posposición adverbial, la prueba chi-cuadrado rechaza la existencia de asociación $\left(\chi^{2}=.294, \mathrm{gl}=2\right.$; $\mathrm{p}=.863){ }^{18}$

\subsection{Según el modo del verbo al que complementan quizá(s) y tal vez}

Como se sabe, quizá(s) y tal vez pueden aparecer con indicativo o con subjuntivo. Según la Nueva gramática de la lengua española (RAEyAALE, 2009, 1955), la elección del modo "depende en gran medida de cómo se interprete la estructura informativa de la oración y, en particular, de que se enfatice o no la verosimilitud de lo que se presenta como probable o la certeza - presente o futura- de lo que aparece como inseguro".

Los resultados que se anotan en las tablas 7 y 8 indican que también hay dependencia entre las variables adverbio y autor tanto en aquellos contextos en los que el verbo se conjuga en indicativo como en subjuntivo $(\mathrm{p}=.000)$. La fortaleza de la relación es ligeramente más alta con indicativo $(\mathrm{V}=.519$ s. $\mathrm{V}=.501)$.

\begin{tabular}{|c|c|c|c|c|c|}
\hline \multirow{2}{*}{ autores } & \multirow{2}{*}{$\mathrm{N}$} & \multicolumn{2}{|c|}{ quizá(s) } & \multicolumn{2}{|c|}{ tal vez } \\
\hline & & $\%$ & RTC & $\%$ & RTC \\
\hline Almudena Grandes & 244 & 65.6 & 2.9 & 34.4 & -2.9 \\
\hline Rosa Ribas & 208 & 65.4 & 2.6 & 34.6 & -2.6 \\
\hline Javier Marías & 644 & 71.3 & 9.5 & 28.7 & -9.5 \\
\hline Eduardo Mendoza & 100 & 43 & -3 & 57 & 3 \\
\hline Isabel Allende & 110 & - & -12.6 & 100 & 12.6 \\
\hline Florencia Bonelli & 80 & 87.5 & 5.6 & 12.5 & -5.6 \\
\hline Eduardo Sacheri & 66 & - & -9.6 & 100 & 9.6 \\
\hline Mario Vargas Llosa & 77 & 9.1 & -8.8 & 90.9 & 8.8 \\
\hline Total & $\begin{array}{c}152 \\
9\end{array}$ & 57.2 & & 42.8 & \\
\hline $\begin{array}{l}\chi^{2}=411.093, g l=7 ; p \\
V=.519\end{array}$ & & & & & \\
\hline
\end{tabular}

Tabla 7. Quizá(s) y tal vez con verbo en indicativo * autores. 


\begin{tabular}{|c|c|c|c|c|c|}
\hline \multirow{2}{*}{ autores } & \multirow{2}{*}{$\mathrm{N}$} & \multicolumn{2}{|c|}{ quizá(s) } & \multicolumn{2}{|c|}{ tal vez } \\
\hline & & $\%$ & RTC & $\%$ & RTC \\
\hline Almudena Grandes & 98 & 45.9 & -1 & 54.1 & 1 \\
\hline Rosa Ribas & 99 & 56.6 & 1.3 & 43.4 & -1.3 \\
\hline Javier Marías & 175 & 64 & 4.2 & 36 & -4.2 \\
\hline Eduardo Mendoza & 51 & 39.2 & -1.7 & 60.8 & 1.7 \\
\hline Isabel Allende & 12 & 8.3 & -3 & 91.7 & 3 \\
\hline Florencia Bonelli & 72 & 90.3 & 7.2 & 9.7 & -7.2 \\
\hline Eduardo Sacheri & 54 & - & -7.8 & 100 & 7.8 \\
\hline Mario Vargas Llosa & 37 & 10.8 & -5 & 89.2 & 5 \\
\hline Total & 598 & 50.7 & & 49.3 & \\
\hline $\begin{array}{l}\chi^{2}=150.158, g l=7 ; p= \\
V=.501\end{array}$ & & & & & \\
\hline
\end{tabular}

Tabla 8. Quizá(s) y tal vez con verbo en subjuntivo * autores.

La diferencia porcentual entre quizá(s) y tal vez cuando acompañan a un verbo en indicativo se asocia a un residuo tipificado significativo en todos los escritores (tabla 7): en Mendoza los casos de tal vez representan el 57\%, lo que pone de manifiesto un incremento de 14.2 puntos con respecto a la frecuencia total.

En cuanto a la combinación de los adverbios con un verbo en subjuntivo (tabla 8), cabe destacar, en primer lugar, que el porcentaje total de quizá(s) solo supera al de tal vez en un $1.4 \%$. Al comparar los porcentajes que se anotan en las tablas 7 y 8 , observamos que los cuatro novelistas españoles aumentan la frecuencia de tal vez cuando utilizan esta locución adverbial con subjuntivo. Asimismo, en este último contexto la locución es la forma con mayor índice de frecuencia en cinco autores (tabla 8): no solo en Allende, Vargas Llosa y Sacheri -quienes, como hemos podido constatar, siempre prefieren tal vez-, sino también en Mendoza (60.8\%) y en Grandes (54.1\%). Sin embargo, los residuos tipificados corregidos muestran valores inferiores a \pm 1.96 en Grandes $( \pm 1)$, Ribas $( \pm 1.3)$ y Mendoza ( \pm 1.7$)$, lo que indica que las variaciones de frecuencias halladas no son significativas. 


\subsection{Según el tiempo del verbo al que complementan quizá(s) y tal vez}

En orden decreciente de acuerdo con el coeficiente de Cramer, las correlaciones significativas entre adverbio y autor ( $\mathrm{p}=.000$ ) muestran una asociación alta (tablas 9, 10, 11 y 12) cuando quizá(s) y tal vez se emplean con un verbo conjugado en presente de indicativo $(\mathrm{V}=.655)$, perfecto simple $(\mathrm{V}=.616)$, presente de subjuntivo $(\mathrm{V}=.565)$ e imperfecto de indicativo $(\mathrm{V}=.524) .{ }^{19}$

\begin{tabular}{|c|c|c|c|c|c|}
\hline \multirow{2}{*}{ autores } & \multirow{2}{*}{$\mathrm{N}$} & \multicolumn{2}{|c|}{ quizá(s) } & \multicolumn{2}{|c|}{ tal vez } \\
\hline & & $\%$ & RTC & $\%$ & RTC \\
\hline Almudena Grandes & 29 & 65.5 & .7 & 34.5 & -.7 \\
\hline Rosa Ribas & 23 & 78.3 & 1.94 & 21.7 & -1.94 \\
\hline Javier Marías & 104 & 78.8 & 5.4 & 21.2 & -5.4 \\
\hline Eduardo Mendoza & 18 & 55.6 & -.3 & 44.4 & .3 \\
\hline Isabel Allende & 15 & - & -4.8 & 100 & 4.8 \\
\hline Florencia Bonelli & 10 & 100 & 2.7 & - & -2.7 \\
\hline Eduardo Sacheri & 26 & - & -6.5 & 100 & 6.5 \\
\hline Mario Vargas Llosa & 9 & - & -3.7 & 100 & 3.7 \\
\hline Total & 234 & 59.4 & & 40.6 & \\
\hline
\end{tabular}

Tabla 9. Quizá(s) y tal vez con verbo en presente de indicativo * autores.

\begin{tabular}{|c|c|c|c|c|c|}
\hline \multirow{2}{*}{ autores } & \multirow{2}{*}{$\mathrm{N}$} & \multicolumn{2}{|c|}{ quizá(s) } & \multicolumn{2}{|c|}{ tal vez } \\
\hline & & $\%$ & RTC & $\%$ & RTC \\
\hline Almudena Grandes & 46 & 71.7 & 2.1 & 28.3 & -2.1 \\
\hline Rosa Ribas & 37 & 67.6 & 1.3 & 32.4 & -1.3 \\
\hline Javier Marías & 80 & 77.5 & 4.3 & 22.5 & -4.3 \\
\hline Eduardo Mendoza & 14 & 42.9 & -1.2 & 57.1 & 1.2 \\
\hline Isabel Allende & 29 & - & -6.7 & 100 & 6.7 \\
\hline Florencia Bonelli & 19 & 89.5 & 2.9 & 10.5 & -2.9 \\
\hline Eduardo Sacheri & 14 & - & -4.5 & 100 & 4.5 \\
\hline Mario Vargas Llosa & 9 & 11.1 & -2.9 & 88.9 & 2.9 \\
\hline Total & 248 & 58.1 & & 41.9 & \\
\hline $\begin{array}{l}\chi^{2}=94.030, g l=7 ; p= \\
V=.616\end{array}$ & & & & & \\
\hline
\end{tabular}

Tabla 10. Quizá(s) y tal vez con verbo en perfecto simple * autores. 


\begin{tabular}{|c|c|c|c|c|c|}
\hline \multirow{2}{*}{ autores } & \multirow{2}{*}{$\mathrm{N}$} & \multicolumn{2}{|c|}{ quizá(s) } & \multicolumn{2}{|c|}{ tal vez } \\
\hline & & $\%$ & RTC & $\%$ & RTC \\
\hline Almudena Grandes & 19 & 52.6 & .1 & 47.4 & -.1 \\
\hline Rosa Ribas & 41 & 48.8 & -.4 & 51.2 & .4 \\
\hline Javier Marías & 120 & 67.5 & $4 \cdot 3$ & 32.5 & $-4 \cdot 3$ \\
\hline Eduardo Mendoza & 41 & 48.8 & -.4 & 51.2 & .4 \\
\hline Isabel Allende & 8 & - & -3 & 100 & 3 \\
\hline Florencia Bonelli & 56 & 87.5 & 5.9 & 12.5 & -5.9 \\
\hline Eduardo Sacheri & 44 & - & -7.3 & 100 & 7.3 \\
\hline Mario Vargas Llosa & 26 & 11.5 & -4.2 & 88.5 & 4.2 \\
\hline Total & 355 & 51.5 & & 48.5 & \\
\hline
\end{tabular}

Tabla 11. Quizá(s) y tal vez con verbo en presente de subjuntivo * autores.

\begin{tabular}{|c|c|c|c|c|c|}
\hline \multirow{2}{*}{ autores } & \multirow{2}{*}{$\mathrm{N}$} & \multicolumn{2}{|c|}{ quizá(s) } & \multicolumn{2}{|c|}{ tal vez } \\
\hline & & $\%$ & RTC & $\%$ & RTC \\
\hline Almudena Grandes & 50 & 70 & 1.86 & 30 & -1.86 \\
\hline Rosa Ribas & 61 & 63.9 & 1.1 & 36.1 & -1.1 \\
\hline Javier Marías & 274 & 71.2 & 6.7 & 28.8 & -6.7 \\
\hline Eduardo Mendoza & 29 & 20.7 & -4.2 & 79.3 & 4.2 \\
\hline Isabel Allende & 36 & - & -7.3 & 100 & $7 \cdot 3$ \\
\hline Florencia Bonelli & 17 & 88.2 & 2.6 & 11.8 & -2.6 \\
\hline Eduardo Sacheri & 11 & - & -3.9 & 100 & 3.9 \\
\hline Mario Vargas Llosa & 30 & 10 & -5.4 & 90 & 5.4 \\
\hline Total & 508 & 57.7 & & 42.3 & \\
\hline
\end{tabular}

Tabla 12. Quizá(s) y tal vez con verbo en imperfecto indicativo * autores.

Como puede observarse, los escritores americanos y también Marías obtienen residuos tipificados significativos en todos los contextos. Por el contrario, los residuos de Ribas no contribuyen al valor del estadístico. Grandes y Mendoza solo muestran una diferencia porcentual asociada a un residuo superior a \pm 1.96 cuando utilizan los adverbios 
como complementos de un verbo conjugado en perfecto simple (tabla 10) o en imperfecto de indicativo (tabla 12), respectivamente; Grandes propicia el uso de quizá(s) (71.7\%, 13.6 puntos por encima del porcentaje total), mientras que Mendoza favorece la aparición de tal vez (79.3\%, lo que supone un incremento del $37 \%$ con respecto a la frecuencia total).

\subsection{Según el segmento fónico al que precedan quizá y quizás}

Los resultados del uso que hacen Ribas, Mendoza y Bonelli de las formas quizá y quizás cuando preceden a una vocal o a una consonante se muestran en las tablas 13 y $14^{20}$.

\begin{tabular}{|c|c|c|c|c|c|}
\hline \multirow{2}{*}{ autores } & \multirow{2}{*}{$\mathrm{N}$} & \multicolumn{2}{|c|}{ quizá } & \multicolumn{2}{|c|}{ quizás } \\
\hline & & $\%$ & RTC & $\%$ & RTC \\
\hline Rosa Ribas & 84 & - & -7.4 & 100 & 7.4 \\
\hline Eduardo Mendoza & 37 & 62.2 & $5 \cdot 7$ & 37.8 & $-5 \cdot 7$ \\
\hline Florencia Bonelli & 58 & 39.7 & 3 & 60.3 & -3 \\
\hline Total & 179 & 25.7 & & 74.3 & \\
\hline \multicolumn{6}{|c|}{$\begin{array}{l}\chi^{2}=60.734, g l=2 ; p=.000 \\
V=.582\end{array}$} \\
\hline
\end{tabular}

Tabla 13. Quizá y quizás ante vocal * autores.

\begin{tabular}{|c|c|c|c|c|c|}
\hline \multirow{2}{*}{ autores } & \multirow{2}{*}{$\mathrm{N}$} & \multicolumn{2}{|c|}{ quizá } & \multicolumn{2}{|c|}{ quizás } \\
\hline & & $\%$ & RTC & $\%$ & RTC \\
\hline Rosa Ribas & 169 & 65.7 & -9.1 & 34.3 & 9.1 \\
\hline Eduardo Mendoza & 96 & 100 & 4.9 & - & -4.9 \\
\hline Florencia Bonelli & 106 & 100 & 5.2 & - & -5.2 \\
\hline Total & 371 & 84.4 & & 15.6 & \\
\hline $\begin{array}{l}\chi^{2}=82.172, g l=2 ; p=. \\
V=.471\end{array}$ & & & & & \\
\hline
\end{tabular}

Tabla 14. Quizá y quizás ante consonante * autores.

Las variables se relacionan de manera significativa $(\mathrm{p}=.000)$ y con una intensidad elevada tanto en el contexto prevocálico $(\mathrm{V}=.582)$ como en el preconsonántico $(\mathrm{V}=.471)$. Asimismo, todos los residuos tipificados corregidos son superiores a \pm 1.96 , lo que indica que las diferencias de frecuencias son significativas. El análisis también revela que Ribas 
evita la forma quizá cuando precede a una palabra que empieza por vocal: el uso de quizás es categórico en el contexto prevocálico y representa el 34.3\% delante de consonante. También el 60.3\% de los ejemplos ante vocal que documentamos en Bonelli corresponden a quizás, pero lo que elude de manera absoluta la autora argentina es el empleo de esta variante delante de consonante. Por último, Mendoza utiliza quizá con mayor frecuencia en ambos contextos: ante vocal el porcentaje se sitúa en el 62.2\% y alcanza el 100\% cuando precede a una consonante.

\section{CONCLUSIONES}

La distribución de los 3476 casos que conforman el corpus objeto de esta investigación indica que quizá(s) tiene una frecuencia más elevada que tal vez $(56.7 \%$ vs. 43.3\%). Ahora bien, la aparición de estas formas adverbiales se ve condicionada por la procedencia, de tal modo que los escritores españoles utilizan más quizá(s) y los americanos prefieren la locución adverbial (65.3\% vs. 70\%). En cuanto a esto último, no puede afirmarse, sin embargo, que se trate de una tendencia absoluta, ya que los datos generales revelan que en la escritora argentina Bonelli el $87.9 \%$ de los ejemplos corresponden a quizá(s). Por otra parte, en Mendoza la diferencia entre quizá(s) y tal vez se reduce a un $4.6 \%$. El análisis estadístico no solo muestra que hay dependencia entre los adverbios de duda y los autores, sino que, además, la fortaleza de la asociación entre estas variables es más alta $(\mathrm{V}=.497)$ que la intensidad hallada al examinar el comportamiento de los adverbios en función de la procedencia ( $\mathrm{V}=.305)$.

También la correlación entre las variables adverbio y autor en los diferentes contextos que hemos considerado arroja resultados significativos. Los datos, ordenados de manera decreciente según la prueba $\mathrm{V}$ de Cramer y los residuos tipificados corregidos, se resumen en la tabla 15. 


\begin{tabular}{|c|c|c|c|c|c|c|c|}
\hline \multirow{2}{*}{ contexto adverbio } & \multirow{2}{*}{ V } & \multirow{2}{*}{$\mathrm{p}$} & \multicolumn{2}{|c|}{ quizá(s) } & \multicolumn{2}{|c|}{ tal vez } & \multirow{2}{*}{$\begin{array}{l}\text { autor con } \\
\text { RTC }<1.96\end{array}$} \\
\hline & & & autor & RTC & autor & RTC & \\
\hline $\begin{array}{l}\text { con presente de } \\
\text { indicativo }\end{array}$ & .655 & .000 & $\begin{array}{l}\text { Marías } \\
\text { Bonelli }\end{array}$ & $\begin{array}{l}5 \cdot 4 \\
2.7\end{array}$ & $\begin{array}{l}\text { Sacheri } \\
\text { Allende } \\
\text { Vargas }\end{array}$ & $\begin{array}{l}6.5 \\
4.8 \\
3.7\end{array}$ & $\begin{array}{l}\text { Grandes } \\
\text { Ribas } \\
\text { Mendoza }\end{array}$ \\
\hline con perfecto simple & .616 & .000 & $\begin{array}{l}\text { Marías } \\
\text { Bonelli } \\
\text { Grandes }\end{array}$ & $\begin{array}{l}4.3 \\
2.9 \\
2.1 \\
\end{array}$ & $\begin{array}{l}\text { Allende } \\
\text { Sacheri } \\
\text { Vargas }\end{array}$ & $\begin{array}{l}6.7 \\
4.5 \\
2.9 \\
\end{array}$ & $\begin{array}{l}\text { Ribas } \\
\text { Mendoza }\end{array}$ \\
\hline $\begin{array}{l}\text { con presente de } \\
\text { subjuntivo }\end{array}$ & .565 & .000 & $\begin{array}{l}\text { Bonelli } \\
\text { Marías }\end{array}$ & $\begin{array}{l}5 \cdot 9 \\
4 \cdot 3\end{array}$ & $\begin{array}{l}\text { Sacheri } \\
\text { Vargas } \\
\text { Allende }\end{array}$ & $\begin{array}{c}7 \cdot 3 \\
4.2 \\
3\end{array}$ & $\begin{array}{l}\text { Grandes } \\
\text { Ribas } \\
\text { Mendoza }\end{array}$ \\
\hline $\begin{array}{l}\text { con imperfecto de } \\
\text { indicativo }\end{array}$ & .524 & .000 & $\begin{array}{l}\text { Marías } \\
\text { Bonelli }\end{array}$ & $\begin{array}{l}6.7 \\
2.6\end{array}$ & $\begin{array}{l}\text { Allende } \\
\text { Vargas } \\
\text { Mendoza } \\
\text { Sacheri }\end{array}$ & $\begin{array}{l}7.3 \\
5.4 \\
4.2 \\
3.9\end{array}$ & $\begin{array}{l}\text { Grandes } \\
\text { Ribas }\end{array}$ \\
\hline con indicativo & .519 & .000 & $\begin{array}{l}\text { Marías } \\
\text { Bonelli } \\
\text { Grandes } \\
\text { Ribas } \\
\end{array}$ & $\begin{array}{l}9.5 \\
5.6 \\
2.9 \\
2.6 \\
\end{array}$ & $\begin{array}{l}\text { Allende } \\
\text { Sacheri } \\
\text { Vargas } \\
\text { Mendoza }\end{array}$ & $\begin{array}{c}12.6 \\
9.6 \\
8.8 \\
3 \\
\end{array}$ & \\
\hline operador oracional & .508 & .000 & $\begin{array}{l}\text { Marías } \\
\text { Bonelli } \\
\text { Ribas }\end{array}$ & $\begin{array}{l}10.5 \\
8.6 \\
2.7\end{array}$ & $\begin{array}{l}\text { Sacheri } \\
\text { Allende } \\
\text { Vargas } \\
\text { Mendoza }\end{array}$ & $\begin{array}{c}12.6 \\
12.5 \\
10.1 \\
3.5 \\
\end{array}$ & Grandes \\
\hline antepuesto al verbo & .508 & .000 & $\begin{array}{l}\text { Marías } \\
\text { Bonelli } \\
\text { Ribas }\end{array}$ & $\begin{array}{c}10.5 \\
8.4 \\
2.4\end{array}$ & $\begin{array}{l}\text { Allende } \\
\text { Sacheri } \\
\text { Vargas } \\
\text { Mendoza }\end{array}$ & $\begin{array}{r}12.5 \\
12.2 \\
9.6 \\
3.5\end{array}$ & Grandes \\
\hline con subjuntivo & .501 & .000 & $\begin{array}{l}\text { Bonelli } \\
\text { Marías }\end{array}$ & $\begin{array}{l}7.2 \\
4.2 \\
\end{array}$ & $\begin{array}{l}\text { Sacheri } \\
\text { Vargas } \\
\text { Allende } \\
\end{array}$ & $\begin{array}{c}7.8 \\
5 \\
3 \\
\end{array}$ & $\begin{array}{l}\text { Grandes } \\
\text { Ribas } \\
\text { Mendoza }\end{array}$ \\
\hline $\begin{array}{l}\text { no es operador } \\
\text { oracional }\end{array}$ & .491 & .000 & $\begin{array}{l}\text { Bonelli } \\
\text { Marías } \\
\text { Grandes }\end{array}$ & $\begin{array}{c}6 \\
5.8 \\
3.3 \\
\end{array}$ & $\begin{array}{l}\text { Vargas } \\
\text { Sacheri } \\
\text { Allende } \\
\end{array}$ & $\begin{array}{c}12.3 \\
9 \\
6.7 \\
\end{array}$ & $\begin{array}{l}\text { Ribas } \\
\text { Mendoza }\end{array}$ \\
\hline
\end{tabular}

Tabla 15. Resumen de datos en los distintos contextos.

La intensidad de la asociación entre las variables es alta en todos los entornos y alcanza un coeficiente más elevado cuando los adverbios complementan a un verbo conjugado en presente de indicativo $(\mathrm{V}=.655)$. De manera sistemática, tres de los cuatro novelistas americanos, Allende, Sacheri y Vargas Llosa, tienen una manifiesta tendencia hacia la locución adverbial, mientras que la autora argentina Bonelli siempre prefiere quizá(s). En cuanto a los escritores españoles, Mendoza es el único en el que la frecuencia 
de tal vez supera a la de quizá(s); concretamente, la diferencia porcentual resulta significativa cuando emplea los adverbios como operadores oracionales antepuestos al verbo y también como complementos de un verbo en indicativo o conjugado en imperfecto. Por otra parte, solo Marías contribuye significativamente al valor del estadístico chi-cuadrado en todos los contextos. En Grandes el valor de los residuos supera el límite de 1.96 cuando los adverbios son complementos de un elemento oracional no verbal, complementos de un verbo en indicativo o de un verbo en perfecto simple; en Ribas cuando utiliza los adverbios como operadores oracionales, antepuestos al núcleo verbal o con un verbo en indicativo.

Por último, al examinar la distribución de las variantes quizá y quizás en función de los autores, también hemos podido constatar una asociación significativa $(\mathrm{p}=.000)$ y de notable intensidad ( $\mathrm{V}=.860$ ). Ribas, Mendoza y Bonelli condicionan la aparición de estas formas ( $\mathrm{p}=.000)$ tanto en el contexto prevocálico $(\mathrm{V}=.582)$ como en el preconsonántico $(\mathrm{V}=.471)$ : la escritora de origen catalán nunca emplea quizá ante vocal, mientras que los otros dos novelistas no usan quizás delante de consonante.

Como conclusión final, podemos señalar que los resultados de este trabajo corroboran estadísticamente que el uso de quizá(s) y tal vez -y también el de quizá y quizás - es un parámetro lingüístico susceptible de ser considerado a la hora de identificar las características idiosincrásicas de un estilo idiolectal.

\section{NOTAS}

1 Para una revisión del concepto de modalidad y sus tipos, véase, entre otros, Otaola Olano, 1988; Jiménez Juliá, 1989; Fuentes Rodríguez, 1991; Carretero, 1991-1992; Gutiérrez Ordóñez, 1996; Martín Zorraquino, 1998; y Ridruejo, 1999.

2 De igual modo, Otaola Olano $(1988,99)$ define la modalidad como "la actitud del sujeto hablante ante el oyente y/o ante el contenido de la predicación emitida por él en el enunciado".

3 Son reforzadores cuando, además del adverbio, aparece alguna marca gramatical que también expresa actitud dubitativa - el modo potencial, frases verbales como deber (de) + infinitivo o la apódosis de una oración condicional-. 
4 En palabras de Otaola Olano $(1988,103)$, "las modalidades lógicas caracterizan la manera en que el sujeto presenta el predicado de la oración como verdadero, contingente (o necesario) o probable (posible)".

5 También computa los casos del adverbio acaso.

6 El número total de ejemplos asciende a 214.

7 Para Martín Zorraquino (1998, 33) el uso de los adverbios de duda "puede depender de los gustos o tendencias del hablante”.

8 La relación de novelas que conforman el corpus se recoge al final de este trabajo.

9 La prueba chi-cuadrado compara las frecuencias observadas con las frecuencias esperadas - esto es, las frecuencias que teóricamente debería haber si no existiera relación entre las variables- para contrastar la hipótesis de que las variables que recoge la tabla de contingencia son independientes o, por el contrario, hay dependencia entre ellas. Si la significación asociada al valor del estadístico es menor que .05, se rechaza la hipótesis de independencia. Por otro lado, como requisito imprescindible para considerar válidos los resultados que arroja la prueba $\chi^{2}$, hemos tenido en cuenta que no aparecieran frecuencias esperadas inferiores a $1 \mathrm{y}$, también, que las frecuencias esperadas menores que 5 no superaran el $20 \%$ del total de casillas de la tabla de contingencia.

10 Los residuos tipificados corregidos se distribuyen con media o y desviación típica 1, por lo que se consideran significativos, con un nivel de confianza del 95\%, los valores absolutos mayores a \pm 1.96 . La relación entre los factores de las variables aumenta a medida que se incrementa el valor del residuo. El signo del residuo es positivo cuando la frecuencia observada es mayor que la frecuencia esperada.

11 Este coeficiente toma valores entre o y 1, por lo que la relación de dependencia entre las variables es más intensa cuanto más se aproxima a 1 el resultado de la prueba. Para la interpretación de los resultados hemos seguido la propuesta que formula Fierro (2010): a) asociación baja: $0<\mathrm{V} \leq .2$; b) asociación media: .2 $<\mathrm{V} \leq .3$; y c) asociación alta: . $3<\mathrm{V} \leq 1$.

12 En cuanto a los resultados que obtiene Bauhr (1990) en su análisis de 11 novelas de 11 autores españoles, ya comentamos que la frecuencia de tal vez (51.9\%) es superior a la de quizá(s), aunque la diferencia no alcanza los 4 puntos porcentuales. La distribución de frecuencias según autor revela que en 6 novelas hay un uso categórico de alguno de los adverbios: cinco autores solo utilizan quizá(s) y otro solo emplea tal vez. Por otro lado, en tres novelistas catalanes predomina la locución adverbial; las ocurrencias documentadas en sus obras representan el 52.3\% del total de casos. Estos datos justifican el ligero incremento que experimenta el porcentaje global correspondiente a tal vez.

13 Un residuo superior en valor absoluto a \pm 2.58 evidencia una relación entre las categorías a un nivel de confianza del $99 \%$.

14 En este punto merece la pena comentar que en Mendoza (2001) y en Ribas (2011) la frecuencia de tal vez supera a la que obtiene quizá(s): en Mendoza (2001) la diferencia solo es de un $5 \%$ $(52.5 \% ; \mathrm{N}=59)$, mientras que en la novela de la autora catalana el porcentaje correspondiente a la locución adverbial representa un importante 90.5\% ( $=126)$-aunque también es muy alta la 
frecuencia de quizá(s) en las tres restantes novelas de Ribas, lo que explica el índice del 63.2\% que recoge la tabla 2-. Así pues, únicamente en estas dos novelas se pone de manifiesto el predominio de tal vez que encuentra Bauhr (1990) en los autores catalanes.

15 Véase González Ollé (1981) y Espejo y Espinosa (2012) para el origen de quizá.

16 Allende y Sacheri no utilizan quizás; solo encontramos 1 ejemplo de quizá en la autora chilena y 4 en el escritor argentino. Este reducido número de ocurrencias hace que en la tabla de contingencia haya 4 casillas (25\%) con frecuencia esperada inferior a 5, motivo por el que estos dos novelistas no aparecen en la tabla 3.

17 Obsérvese, de manera concreta, que en Mendoza y en Allende todos los adverbios utilizados como operadores preceden al verbo.

18 En esta fase del análisis solo hemos podido incluir los casos encontrados en las novelas de Grandes, Ribas y Marías. En Bonelli, Sacheri y Vargas Llosa la frecuencia esperada es inferior a 5 en las 6 casillas (50\%) de la tabla de contingencia.

19 El análisis con el resto de tiempos verbales no arroja una probabilidad estadísticamente significativa o bien no resulta fiable porque las frecuencias esperadas inferiores a 5 superan el límite establecido del 20\%.

20 Hemos prescindido del resto de escritores porque solo utilizan una de las variantes. Como ya vimos (nota 16 y tabla 3), Marías, Allende y Sacheri solo usan quizá, mientras que Vargas Llosa únicamente emplea quizás. De igual modo, tampoco hemos incluido a Grandes porque en sus novelas solo encontramos un caso de quizá. Por otro lado, también hemos excluido del análisis el entorno prepausal ya que las frecuencias esperadas inferiores a 5 superaban el $20 \%$.

\section{REFERENCIAS BIBLIOGRÁFICAS}

AUROVÁ, M. 2013. "El uso del subjuntivo/indicativo con el operador de modalidad quizá(s): análisis del corpus", Écho des études romanes, 9 (1), 19-33.

BALLY, CH. 1932. Linguistique générale et linguistique française. Berne: A. Francke.

BAUHR, G. 1990. “Notas sobre el uso de ‘quizá(s)' y ‘tal vez’ en un corpus de español moderno: ONE71”, Anales, 2, 177-193.

CARRETERO, M. 1991-1992. "Una propuesta de tipología de la modalidad: la aceptación como categoría modal”, DICENDA, Cuadernos de Filología Hispánica, 10, 41-61.

ESPEJO MURIEL, M. M. y ESPINOSA ELORZA, R. M. 2012. “Quiçab, quiçá, quizá”, en Montero Cartelle, E. y Manzano Rovira, C. (eds.), Actas del VIII Congreso Internacional de Historia de la Lengua Española. Madrid: Meubook, 749-76o. 
FIERRO, J. 2010. Análisis estadístico univariado, bivariado y variables control. Disponible en: http://chitita.uta.cl/cursos/2012-1/oooo104/recursos/r-25.pdf [Consultado 21 enero 2015].

FUENTES RODRÍGUEZ, C. 1991. “Algunas reflexiones sobre el concepto de modalidad”, Revista de la Sociedad Española de Lingüística Aplicada, 7, 93-108.

GONZÁLEZ OLLÉ, F. 1981. “Evolución de los grupos d's y t’s y nueva etimología de quizá”, en Mélanges de philologie et de toponymie romans offerts à Henri Guiter. Perpignan: Imprimerie Catalane, 309-318.

GUTIÉRREZ ORDÓÑEZ，S. 1996. "La periferia verbal (II): complementos del verbo enunciativo y atributos de modalidad”, en Briz, A, Gómez, J., Martínez, M. J. y grupo Val.Es.Co (eds.), Pragmática y gramática del español hablado. Actas del II Simposio sobre análisis del discurso oral. Valencia-Zaragoza: Universidad de Valencia-Pórtico, 91-108.

JIMÉNEZ JULIÁ, T. 1989. “Modalidad, modo verbal y modus clausal en español”, Verba, 16, 175-214.

KOVACCI, O. 1999. “El adverbio”, en Bosque, I. y Demonte, V. (dirs.), Gramática descriptiva de la lengua española. Madrid: Espasa Calpe, 705-786.

LYONS, J. 1977. Semantics. Cambridge: Cambridge University Press.

MARTÍN ZORRAQUINO, M. A. 1998. "Aspectos de la gramática y de la pragmática de las partículas de modalidad en español actual”, en Jiménez Juliá, T., Losada Aldrey, M.C. y Márquez Caneda, J. F. (eds.), Español como lengua extranjera: enfoque comunicativo y gramática. Actas del IX Congreso Internacional de ASELE. Santiago de Compostela: Universidade de Santiago de Compostela, 25-56.

OTAOLA OLANO, C. 1988. "La modalidad (con especial referencia a la lengua española)", Revista de Filología Española, 68 (1-2), 97-117.

PALMER, F. R. 1986. Mood and Modality. Cambridge: Cambridge University Press.

PERKINS, M. R. 1983. Modal Expressions in English. Londres: Frances Pinter.

REAL ACADEMIA ESPAÑOLA. 2014. Diccionario de la lengua española (DRAE). Madrid: Espasa.

REAL ACADEMIA ESPAÑOLA Y ASOCIACIÓN DE ACADEMIAS DE LA LENGUA ESPAÑOLA (RAEyAALE). 2005. Diccionario panhispánico de dudas (DPD). Madrid: Santillana. . 2009. Nueva gramática de la lengua española. Madrid: Espasa. 
RIDRUEJO, E. 1999. "Modo y modalidad. El modo en las subordinadas sustantivas", en Bosque, I. y Demonte, V. (dirs.), Gramática descriptiva de la lengua española. Madrid: Espasa Calpe, 3209-3251.

WOEHR, R. 1972. “'Acaso', 'quizá(s)', 'tal vez': Free Variants?”, Hispania, 55 (2), 320-327.

ZAVADIL, B. 1968. "Medios expresivos de la categoría de modalidad en español", Ibero-Americana Pragensia, 2, 57-86.

\section{ANEXO}

\section{NOVELAS QUE COMPONEN EL CORPUS}

Allende, Isabel:

Inés del alma mía. 2006.

La isla bajo el mar. 2009.

El cuaderno de Maya. 2011.

El juego de Ripper. 2014.

Bonelli, Florencia:

Marlene. 2003.

Lo que dicen tus ojos. 2006.

El cuarto arcano. 2007.

Me llaman Artemio Furia. 2009.

Grandes, Almudena:

Los aires difíciles. 2002.

Castillos de cartón. 2004.

El corazón helado. 2007.

Inés y la alegría. 2010.

Marías, Javier:

Tu rostro mañana. 1. Fiebre y lanza. 2002.

Tu rostro mañana. 2. Baile y sueño. 2004.

Tu rostro mañana. 3. Veneno y sombra y adiós. 2007.

Los enamoramientos. 2011. 
Mendoza, Eduardo:

La aventura del tocador de señoras. 2001.

Mauricio o las elecciones primarias. 2006.

Riña de gatos. Madrid 1936. 2010.

El enredo de la bolsa y la vida. 2012.

Ribas, Rosa:

El pintor de Flandes. 2006.

Entre dos aguas. 2007.

Con anuncio. 2009.

En caída libre. 2011.

Sacheri, Eduardo:

Aráoz y la verdad. 2008.

El secreto de sus ojos. 2009.

Papeles en el viento. 2011.

Ser feliz era esto. 2014.

Vargas Llosa, Mario:

El paraíso en la otra esquina. 2003.

Travesuras de la niña mala. 2006.

El sueño del celta. 2010.

El héroe discreto. 2013. 